\title{
Short-wavelength Raman spectroscopy for in-situ water analysis: a feasibility study
}

\section{Andrea Sterzi, Olga Sambalova, Urs Schneider, Davide Bleiner, Andreas Borgschulte}

Andrea Sterzi, Olga Sambalova, Urs Schneider, Davide Bleiner, Andreas Borgschulte, "Short-wavelength Raman spectroscopy for in-situ water analysis: a feasibility study," Proc. SPIE 11886, International Conference on X-Ray Lasers 2020, 118860Y (8 July 2021); doi: 10.1117/12.2596045 


\title{
Short-wavelength Raman Spectroscopy for in-situ Water Analysis: A Feasibility study
}

\author{
Andrea Sterzi ${ }^{\mathrm{a}}$, Olga Sambalova ${ }^{\mathrm{a}, \mathrm{b}}$, Urs Schneider ${ }^{\mathrm{c}}$, Davide Bleiner ${ }^{\mathrm{a}, \mathrm{b}}$, and Andreas \\ Borgschulte $\mathrm{a}^{\mathrm{a}, \mathrm{b}}$ \\ ${ }^{a}$ Laboratory for Advanced Analytical Technologies, Empa - Swiss Federal Laboratories for \\ Material Science and Technology, Überlandstrasse 129, CH-8600 Dübendorf, Switzerland \\ ${ }^{b}$ Department of Chemistry, University of Zurich, Winterthurerstrasse 190, CH-8057 Zürich, \\ Switzerland \\ ${ }^{\mathrm{c}}$ Rascope ag, Espenstrasse CH-135, Widnau, Switzerland
}

\begin{abstract}
Short-wavelength $(\lambda<260 \mathrm{~nm})$ Raman spectroscopy offers an advantage of a generally higher sensitivity than Raman spectroscopy in the visible range. An application with high potential is its use for environmental water analysis targeting archetypal compounds that are present in industrial and urban sewage water. However, this application is feasible only if specific performance benchmarks are met. We validate the applicability of a simple and cost-effective deep-UV Raman spectrometer $\left(\lambda_{e x c}=236.5 \mathrm{~nm}\right)$. The analysis brings to the fore that the experimentally derived detection limits the studied archetypal compounds are to high by several orders of magnitude. We outline potential further development and associated limitations. These are the deterioration of the analysed species by photolysis as a consequence of the high photon energy and intensity, and the self absorption of the UV radiation. These effects are explained and partially corrected along a simple mathematical model from which a general limit of detection is estimated.
\end{abstract}

Keywords: Deep-UV Raman spectroscopy, organic pollutants, self-absorption, water quality sensor

\section{INTRODUCTION}

Clean water is an essential and one of the most important aspects of the United Nations sustainable development goals. ${ }^{1}$ However, water pollution is diminishing the accessibility to clean water worldwide. Apart from long known fertilizer-derived compounds, such as nitrate $\mathrm{NO}_{3}^{-}$and nitrite $\mathrm{NO}_{2}^{-}$ions, ${ }^{2,3}$ new threats are posed by the appearance of so-called Contaminants of Emerging Concern (CECs), which are difficult to remove from effluent streams. Among them, antidepressant agents Citalopram (CIT), Venlafaxine (VEN) and Carbamazepine (CBZ) and a widely used corrosion inhibitor, Benzotriazole, are in the spotlight. ${ }^{4}$ Several studies report their concentration levels in waste water in the range of tens of $\mu \mathrm{g} / \mathrm{l}$, leading to serious threats for the aquatic fauna and plants. ${ }^{5-8}$ In the case of nitrate ions, the maximum reported contamination levels is higher, approx. $\leq 50$ $\mathrm{mg} / \mathrm{l}$, according to the World Health Organization (WHO). ${ }^{9-11}$ Even more harmful are nitrite ions, which can be formed via photolysis process under UV illumination. ${ }^{11-14}$ These compounds in waste water are normally measured via liquid chromatography - mass spectrometry (LC-MS). ${ }^{5-7}$ The technique provides high sensitivity (beyond $\mu \mathrm{g} / \mathrm{l}$ ) and chemical selectivity. ${ }^{15}$ However, the process has to be performed in a chemical laboratory and requires intermediate steps (e.g. sample preparation and separation of the elemental components). In addition, the instrumentation cost and dimension renders this technique unsuitable for on-line detection.

Optical spectroscopy may be a valid on-line alternative. Optical vibrational spectroscopy has high potential, because the spectra resemble a molecular fingerprint. Infrared gas analysis is known to detect trace impurities down to ppb level. ${ }^{16}$ Infrared spectroscopy is not applicable to water analysis because of the large absorption in this wavelength range. An optical water analysis method must use the water window from around $1 \mu \mathrm{m}$ to 200 $\mathrm{nm},{ }^{17}$ a criterion, which is met by Raman spectroscopy. However, despite its high versatility, it suffers from an intrinsically low sensitivity. In fact, the intensity of the Raman scattered light $I$ is several orders of magnitude

Send correspondence to A. Borgschulte: E-mail: andreas.borgschulte@empa.ch, Telephone: +41 587654639

International Conference on X-Ray Lasers 2020, edited by Davide Bleiner,

Proc. of SPIE Vol. 11886, 118860Y · @ 2021 SPIE - CCC code:

0277-786X/21/\$21 doi: $10.1117 / 12.2596045$

Proc. of SPIE Vol. 11886 118860Y-1 


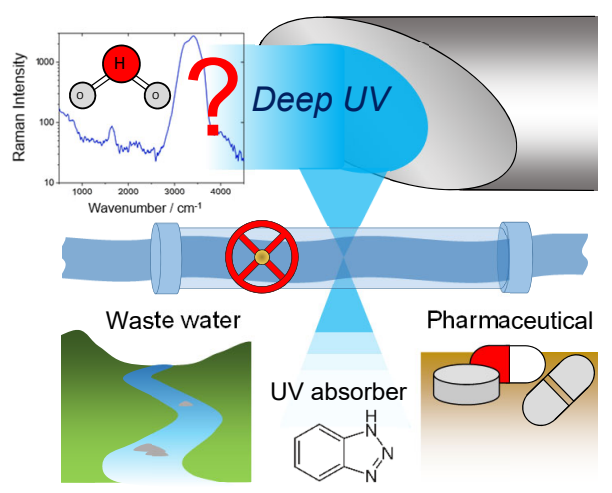

Figure 1. Deep-UV Raman spectroscopy for monitoring water quality and its potential applications.

lower than the excitation light $I_{0}$, being approximately one Raman photon scattered for every $10^{-6}$ incident photons. Another substantial limiting factor is fluorescence, which is often dominant in organic samples and overlaps with the Raman bands. ${ }^{18}$ Several approaches have been proposed to enhance the signal while limiting fluorescence, e.g. taking advantage of new technologies in the field of laser sources, time-resolved detection, optical components, devices, sensors and plasmonic substrates. ${ }^{18-21}$ Summarizing, Raman spectroscopy in the deep-UV energy range fulfils the above given criteria. Its main advantage is the confinement of Raman Stokes lines in the region up to $4000 \mathrm{~cm}^{-1}$ ( $\Rightarrow \lambda \simeq 260 \mathrm{~nm}$ ), well separated from the typical fluorescence emission $(\lambda>260$ $\mathrm{nm}) .{ }^{22,23}$ The Raman signal, $I$, is inversely proportional to the forth power of the wavelength $\left(I \propto \lambda_{\text {exc }}^{-4}\right.$, hence it is markedly enhanced by shortening the latter. Further signal enhancement can be reached, if the photon energy is resonant with a specific electronic transition, the so-called resonant Raman spectroscopy (RRS), which is very likely to occur in the UV range (UV-RRS). Based on this selective enhancement mechanism, UV-RRS has been exploited to study several molecular species showing electronic absorption bands in the UV spectral range, e.g. nucleic acids, proteins and drugs. ${ }^{24,25}$ Recently, this method proved its applicability also in the field of water analysis on example of antibiotics in solutions and urine. ${ }^{26}$ This pioneering study by C. Domes et al. reports the detection by deep-UV RRS of Moxiflacin, Meropenem and Peperacilin in aqueous media with a limit of detection (LOD) in the order of $5 \mathrm{mg} / \mathrm{l}$. Nitrate and nitrite ions in waste water have been more extensively studied, A. Ianoul et al. ${ }^{27}$ reached a LOD of about $1 \mathrm{mg} / \mathrm{l}(14 \mu \mathrm{M})$ for $\mathrm{NO}_{3}^{-}$and $\mathrm{NO}_{2}^{-}$. Later on, several works reported Raman detection of nitrite ${ }^{28,29}$ while focusing on the UV light induced photo-degradation, e.g. photolysis of nitrate to form nitrite ions, another "side-effect" correlated to the use of highly energetic photons. ${ }^{12-14,30,31}$

Despite such remarkable results, the application of deep-UV Raman as a standard analytical method to perform routine water analysis is still limited. Several technical factors restrict its use. First, the mere generation of deep-UV light requires customized high power laser sources such as Ti:Sapphire oscillators, Nd:YAG (4th and 5 th harmonics at 266 and $213 \mathrm{~nm}$ ), CW intracavity frequency-doubled argon or kripton ion lasers (lines at 257, 248, 244, $229 \mathrm{~nm}$ ), Raman-shifted third harmonics in gas from Nd:YAG (204 nm) or diode-pumped solid state (DPSS) Nd:YVO4 $(213 \mathrm{~nm}) \cdot{ }^{26,32-34}$ Their cost and complexity is also often accompanied with a lack of mobility. Alternatively, hollow cathode lasers (e.g. NeCu pulsed laser emitting at $248.5 \mathrm{~nm}$ ) are more affordable and have recently found applications in aerospace, being used for performing in-situ minerals investigation. ${ }^{35}$ However, their lifetime is relatively short and their peculiar annular beam profile may impede a proper beam focusing. Tunable light sources covering the deep-UV range exist in the form of tunable lasers ${ }^{31,36}$ and UV synchrotron radiation, ${ }^{37}$ enabling measurement of analytes in the resonance condition. However in both cases they cannot be implemented in view of developing a portable device. Furthermore, the collection of the Raman signal from a multi-wavelength light source, i.e. the separation of the Rayleigh from the Raman light, is even more challenging. ${ }^{31}$ Additional deep-UV range effects potentially include optical self-absorption, photo-decomposition of the sample and resonances, practically affecting the measured spectrum. Those "intrinsic-processes", which are seldom present in standard Raman spectroscopy, while boosting the sensitivity (resonance enhancement) increase the complexity of the measurement and thus impede an extensive application.

In this work we evaluate a compact and cost-effective deep-UV Raman spectrometer for in-situ environmental analysis, i.e. detection of archetypal contaminants in aqueous environment at $\mathrm{mg} / \mathrm{l}$ concentrations (sketched 
in Fig.1). We investigate advantages and limitations of the quasi-resonance approach on different pollutant categories: antidepressant agents, corrosion inhibitor and fertilizer-derived compounds. Specifically, the following analytes are measured: Citalopram, Venlafaxin, Carbamazepine, Benzotriazole in solutions, nitrate and nitrite ions in deionized (pure) and environmental (river) water. We provide a reliable LOD and asses effects such as self-absorption, fluorescence and photolysis, which selectively affect the acquired spectra. The deep-UV Raman spectrometer built in our lab is based on recent technological advances in deep-UV micro-chip laser and optical components. A direct comparison between visible (commercial Raman, $\left.\lambda_{e x c}=532 \mathrm{~nm}\right)$ and deep-UV $\left(\lambda_{e x c}=\right.$ $236.5 \mathrm{~nm}$ ) was made for all samples under investigation. The impact of the self-absorption, i.e. an induced non-linear dependence between the Raman intensity and the analyte concentration, is investigated. From this, the effective laser beam absorption length through the sample is estimated. Although the strong attenuation of laser and scattered light common for strong UV absorbers, such as benzotriazole, hampers the analysis, we show how the effect can be used to estimate the overall molar concentration of even strongly UV-absorbing compounds. This important analysis parameter (attenuation) is already used to perform on-line analysis in sewage water plants. As Raman sensitivity of compounds is often not known in advance and is low, determined by the scattering cross sections, the simultaneous acquisition of Raman and attenuation parameters paves the way for the automatic (computerized) evaluation of multi-component systems.

\section{METHODOLOGY}

\subsection{Deep-UV Raman setup}

This study is based on the prototype deep-UV Raman spectrometer described in Ref. ${ }^{31}$ The system is further reduced in dimensions and cost and is adapted for measurements of liquid samples in $180^{\circ}$ backscattering geometry (Fig. 2). A commercial, passively q-switched microchip laser (Pulselas-P-236 by Alphalas) emitting $5 \mathrm{~mW}$ @ $\lambda_{\text {exc }}=236 \mathrm{~nm}$, with $10 \mathrm{KHz}$ repetition rate is used as a light source. A single element (BS/filter in Fig. 2) reflects the incident light wavelength, while transmitting the Stokes line. Thereby, the laser line is removed. A single aluminum $90^{\circ}$ off-axis parabolic mirror is used to focus the beam and collect the scattered light. ${ }^{31}$ The complete instrument with the compact light source fits on a $45 \times 30 \mathrm{~cm}$ breadboard. The advantages of the chosen excitation wavelength include a spectral separation of the fluorescence and Raman signals and availability of the optical components. ${ }^{23}$ In particular, commercial Rayleigh Raman filters (e.g., from Semrock) are available for the chosen wavelength and not for shorter excitation wavelength, e.g. Nd:YAG based $213 \mathrm{~nm}$. The signal loss due to the absorption by optical components is negligible. The effective laser beam power at the sample position is reduced to less than $40 \%$ (from 5 to $3 \mathrm{~mW}$ ). Due to its simplicity, the presented optical scheme is adaptable to further modifications without requiring the complete re-design of the set-up.

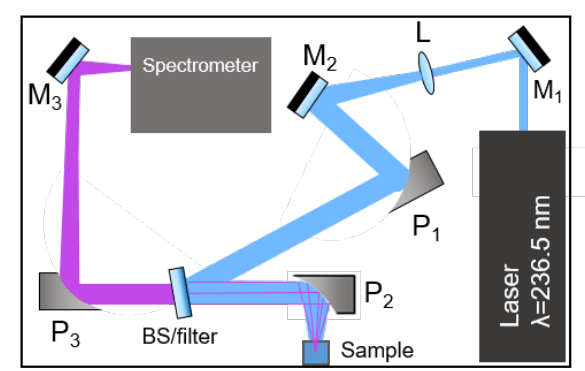

Figure 2. Simplified optical scheme of the deep-UV Raman spectrometer. The optical components include UV-mirrors $(\mathrm{M})$, a diverging lens (L), off axis parabolic mirrors $(\mathrm{P})$ and a beam splitter/edge filter (BS/filter). The laser beam is shown in blue while the scattered light is shown in purple.

\subsection{Raman spectroscopy in the visible energy range}

Visible Raman spectra were measured by meas of a continuous wave laser (Ocean Optics, $100 \mathrm{~mW}$ nominal power, $40 \mathrm{~mW}$ effective power at the focal point, $\lambda_{e x c}=532 \mathrm{~nm}$ ) and a Raman probe (Bruker) connected by an optical fiber. All Raman spectra were acquired with the integration time of 20 seconds and 100 averages. 
The dark spectrum (camera noise) was subtracted with no additional smoothing procedure. As a threshold to perform peak identification during the spectra evaluation, we assumed a signal to noise ratio equal to 3 .

\subsection{Ion Chromatography and UV absorption spectroscopy}

As a validation method, Ion Chromatography (IC) was adopted to determine the nitrate content in the sample withdrawn from the river. The measurements were performed on a Metrohm IC 882 instrument with a ASUPP 5-250 separation column. Certified standard reference materials were used. UV absorption measurements were performed on a UV spectrometer (Varian UV scan 50 BIO).

\subsection{Samples}

Pharmaceuticals and benzotriazole were purchased from Sigma-Aldrich, specifically: Citalopram hydrobromide $\left(\mathrm{C}_{20} \mathrm{H}_{21} \mathrm{FN} \mathrm{N}_{2} \mathrm{O} \cdot \mathrm{HBr}\right.$, molecular weight $\left.=405.3 \mathrm{~g} / \mathrm{mol}\right)$; Venlafaxine hydrochloride $\left(\mathrm{C}_{17} \mathrm{H}_{27} \mathrm{NO}_{2} \cdot \mathrm{HCl}\right.$, molecular weight $=313.8 \mathrm{~g} / \mathrm{mol})$; Carbamazepine $\left(C_{15} H_{12} N_{2} O\right.$, molecular weight $\left.=236.27 \mathrm{~g} / \mathrm{mol}\right)$, benzotriazole $\left(C_{6} H_{5} N_{3}\right.$, molecular weight $=119.1 \mathrm{~g} / \mathrm{mol}$ ). The structure of each molecule is reported in Fig. S1 Supplemental Material. Solutions were prepared using deionized water and diluted to required concentration. Nitrate solutions were prepared in deionized water and an environmental sample was taken from a local river (Chriesbach, Dübendorf Switzerland). Samples were stored at 5 degrees Celsius and were measured in quartz cuvettes.

\section{THEORY}

\subsection{Excitation and relaxation processes in the deep-UV spectral range}

The interaction of a Raman-active molecule with the incident photon depends on the laser wavelength. The physical origin of such wavelength-dependent behaviour is briefly schematically represented in an extended Jablonsky diagram (Fig. 3). Upon non-resonant irradiation the excitation frequency $\nu_{0}$ is far from inducing any electronic transition, hence the molecule is excited from the ground state (GS) to a virtual state (Fig. 3A). ${ }^{24}$ Radiation is emitted back at the same frequency $\nu_{0}$ (Rayleigh elastic scattering) and via inelastic scattering at a shifted frequency $\nu \pm \nu_{0}$ (Stokes and Anti-Stokes inelastic scattering), which corresponds to the vibrational or rotational energies. In Fig. 3, for simplicity, only the the Stokes inelastic scattering $\left(\nu-\nu_{0}\right)$ is represented. This phenomenon is known as Standard Raman (or non-Resonant Raman) and virtually occurs at any incident photon wavelength. ${ }^{38}$

When the photon energy of the excitation source is equal to the energy of an electronic transition, the molecule is excited from the GS to a real electronic state (Fig. 3B). In such resonance condition, the scattered signal can be enhanced of several order of magnitude (up to $10^{8}$ times higher) when compared to standard Raman scattering. ${ }^{22}$ The enhancement is due to the resonance-increased probability of excitation compared to an excitation to a virtual state. ${ }^{25}$ The signal is also enhanced in quasi-resonance condition, and increases as the photon energy approaches the electronic transition. The emission of Stokes and anti-Stokes light is the fastest relaxation channel with competing emission originating from fluorescence (Fig. 3C). Fluorescence takes place on longer time scale than Raman scattering (nanosecond timescale) and often obstructs the weaker Raman signal. ${ }^{22}$ Additionally, if the molecule undergoes a process of photolysis, molecular bonds are broken (Fig. 3D). This leads to the formation of new compounds, which then act as Raman-active light scattering molecules.

The majority of molecular species exhibit absorption bands in the UV and deep-UV spectral range ${ }^{25}$ hence the scenario depicted in cases (B,C,D) is expected when deep-UV light is used to excite the specimen. We notice that the molecules under investigation do not exhibit absorption bands precisely at the excitation wavelength (236.5 nm; Figs.S5 and S6). Therefore, our method of choice is quasi-resonant deep-UV Raman Spectroscopy.

\subsection{Raman intensity and self-absorption}

The sensitivity of Raman analysis is mainly determined by the Raman scattering cross section, $\sigma$. The scattering is much stronger in resonant Raman, as $\sigma$ increases proportionally to the square of the molar absorptivity, $\varepsilon$, of the absorber at the resonant frequency $\nu_{0}:{ }^{39}$

$$
\sigma \propto \nu^{4} \varepsilon^{2}\left(\nu_{0}\right)
$$




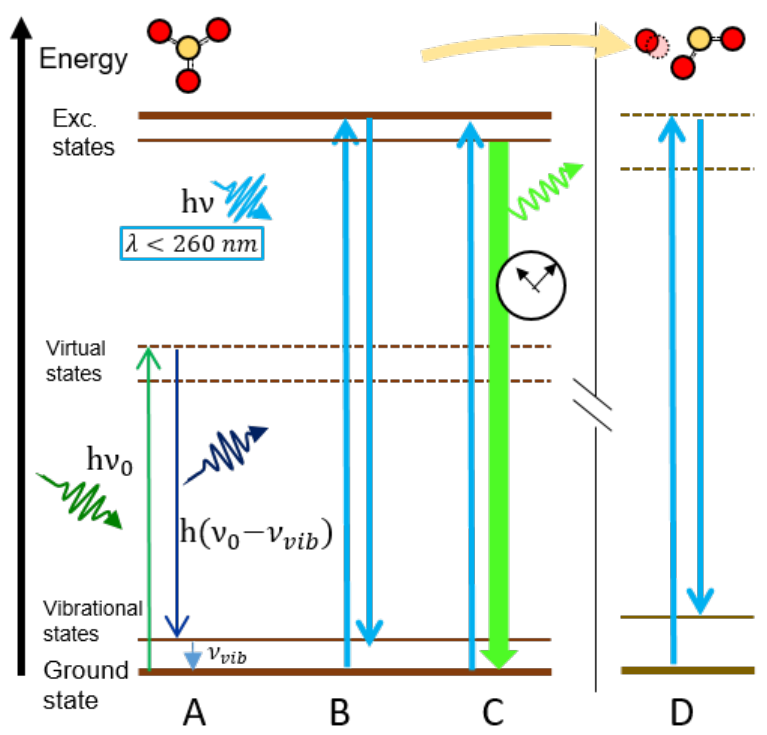

Figure 3. Extended Jablonsky diagram showing main possible quantum electronic transitions occurring at different excitation wavelengths. (A) Standard Raman in the visible range, (B) Resonant Raman (C) Photon absorption and time delayed (clock) fluorescence emission (D) Photolysis generating new compound exciting new Raman emissions. The broken line separates the system before and after the photolysis. Second order processes and the probability of each electronic transition are omitted for simplicity.

The values of $\sigma$ can be as large as $10^{-22} \mathrm{~cm}^{-2} \cdot \mathrm{mol}^{-1} \cdot \mathrm{sr}^{-1}$, which is roughly $10^{8}$ times higher than in non-resonant Raman $\left(\approx 10^{-30} \mathrm{~cm}^{-2} \cdot \mathrm{mol}^{-1} \cdot \mathrm{sr}^{-124}\right)$. Practically, effective sensitivity depends also on various parameters: the collection optics, scattering geometry, excitation wavelength, laser power, etc. In short-wavelength Raman the factor determining Raman sensitivity is the phenomenon of differential self-absorption. Briefly, the excitation light is attenuated by passing through the sample (self-absorption), affecting the collected Raman signal. The scattered light travelling back through the sample is also self-absorbed, but the single Raman lines are differently attenuated due to the dependency of $\varepsilon$ (molar absorptivity) on the frequency of the scattered radiation (differential self-absorption). ${ }^{39-41}$ Several theoretical and optical models have been proposed to describe the intensity of Raman bands accounting for experimental conditions and considering the differential self-absorption phenomenon (mostly analyte-related). ${ }^{35,39-41}$ It is not the scope of this work to introduce a new model or discuss in detail the existing ones, but rather to evaluate, in a simplified way, the impact of differential self-absorption on the measured Raman spectra (we refer to it from here as self-absorption) by adapting the model introduced by Shiver et al. ${ }^{40,41}$

The intensity of a specific Raman band $i$, quantified in counts during the measurement process, can be generally expressed by:

$$
I_{i}=k \sigma_{i} N_{i}
$$

where $k$ is a coefficient accounting for the experimental conditions, i.e. laser Intensity (here defined as $W / \mathrm{cm}^{2}$, illuminated volume $\mathrm{V}$, collection aperture and detector efficiency, $\sigma_{i}$ is the scattering cross section and $N_{i}$ is the number of analyte molecules per unit of volume. In order to quantify $I_{i}$, the intensity of the Raman scattered light at a specific wavelenght, $I_{i, R}$, is estimated as follows. During the measurement process the Raman light is converted by the spectrometer, through a conversion factor $k_{s}$ :

$$
I_{i}=k_{s} I_{i, R}
$$

We define $V$ as the interacting volume of the focused laser beam with the liquid sample. $V$ can be roughly approximated by a portion $L$ of a Gaussian beam propagating along the $\mathrm{z}$ direction (Fig. 4).

The following approximations are considered: 1) the scattered light, which may originate outside of the interacting volume is neglected and 2) the detector collects homogeneously the radiation scattered from different 


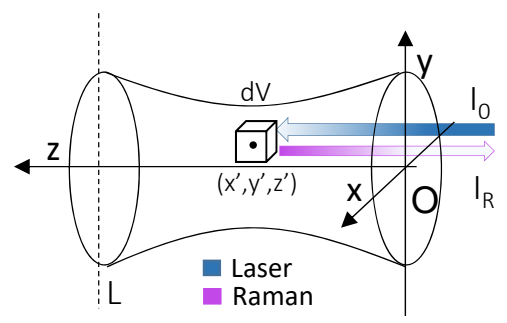

Figure 4. Illuminated Gaussian-like volume in back-scattering geometry and self-absorption. Laser and scattered light propagates from the origin $\mathrm{O}$ to the point ( $\left.\mathrm{x}^{\prime}, \mathrm{y}^{\prime}, \mathrm{z}^{\prime}\right)$. L is the depth of focus.

point inside the interacting volume. The infinitesimal scattering element $d V$ at the position ( $\left.\mathrm{x}^{\prime}, \mathrm{y}^{\prime}, \mathrm{z}^{\prime}\right)$, stems for a generic sampling point which is excited by the laser and which scatters light backwards. The laser light distribution function, $I_{\lambda}$, is attenuated along the $\mathrm{z}$ direction according to the Beer Lambert's law and expressed by:

$$
I_{\lambda}=I_{0}\left(x^{\prime}, y^{\prime}, z^{\prime}\right) e^{-\alpha z}
$$

where the nominal intensity of the laser is $I_{0}$ and $\alpha\left(\mathrm{mm}^{-1}\right)$ is the absorption coefficient at the excitation wavelength $\lambda$. The Raman scattered light is also attenuated according to the Beer Lambert's law, and the loss term is $\exp \left(-\beta \frac{z}{\cos \theta}\right)$, where $\beta$ is the absorption coefficient at the Raman band wavelength. The solid scattering angle $\Omega$ is relatively small in our setup and each scattering point $d V$ scatters over a negligible angle $d \theta$, hence $\cos \theta \approx 1$. By defining $I_{\Omega}$ as its distribution function, the fraction of total scattered light is expressed by $k_{\Omega}=\int I_{\Omega} d \Omega$ over the solid angle $\Omega$. The overall expression for $I_{i, R}$, accounting for all the introduced terms is:

$$
I_{i, R}=k_{\Omega} \iiint_{V} \sigma_{i} N_{i} I_{0}(x, y, z) e^{-\alpha z} e^{-\beta z} d x d y d z
$$

The terms $\sigma_{i}, N_{i}$ do not depend on the spatial coordinates and $P$ (Watt) is the laser power without any absorption loss at a surface centered at $(\mathrm{x}, \mathrm{y})$ along $\mathrm{z}$ direction, estimated as the integral of the laser nominal intensity $P=\iint I_{0}(x, y) d x d y$. By incorporating the constant $k_{s}$ of eq.(3), we obtain the expression for the detected intensity:

$$
I_{i}=k_{s} k_{\Omega} P \sigma_{i} N_{i} \int_{0}^{l} e^{-\alpha z} e^{-\beta z} d z
$$

The integral is calculated over the length $l$, namely effective absorbance length. The solution and final expression for $I_{i}$ is then:

$$
I_{i}=\left(k_{s} k_{\Omega} \sigma_{i} P\right)\left(\frac{N_{i}}{\alpha+\beta}\right)\left(1-e^{-l(\alpha+\beta)}\right)
$$

This equation was originally formulated to evaluate self-absorption in mixtures containing resonantly active components. ${ }^{41}$ Here, we make use of it by considering the solution as a mixture of resonant (analyte) and nonresonant (water) components. In non-resonant condition $(\alpha+\beta)$ is small $(<1)$. The term $\left(1-e^{-(\alpha+\beta) l}\right) \div(\alpha+\beta) \approx$ 1 , so in eq.(7) the intensity $I_{i, R} \propto N_{i}$, hence proportional to the analyte concentration. The length $l$, in absence of absorption, coincides with $L$ as the collected light is scattered from the entire illuminated volume $V$. Conversely, in resonance (or pre-resonance) $(\alpha+\beta)>1$ and the exponential $\left.e^{-(\alpha+\beta) l}\right)<<1$, meaning that the beam is extensively absorbed along the probed volume. In this case, $\left(1-e^{-(\alpha+\beta)} l\right)$ is $\approx 1$ and the second bracket of eq.(7) is constant, as both $N_{i}$ and the denominator are proportional to the concentration $c\left(\alpha=\varepsilon_{\alpha} \cdot c\right.$ and $\varepsilon_{\alpha}$ is the molar absorption coefficient). A strong attenuation of the non-resonant component, i.e. a water Raman mode, is expected. In this respect we define the inhibition coefficient $K_{i}$ as the ratio of the inhibited and non-inhibited water mode $I_{\text {water }} \div I_{0, \text { water }}$. This term, as derived from eq.(7), results in the following relation:

$$
K_{i}:=\frac{I_{\text {water }}}{I_{0, \text { water }}}=\frac{1-e^{-l(\alpha+\beta)}}{l(\alpha+\beta)}
$$


Water peak intensities are taken from Raman spectra while $\alpha$ and $\beta$ are obtained from the UV absorption measurements through $\alpha=A b s \div 10 \mathrm{~mm} \cdot \ln (10)$, where Abs is the measured Absorbance. The term $l$ is estimated by graphically solving eq.(8). It is worth noticing that neglecting the effective laser intensity distribution and the spectrometer clipping effect represent a strong approximation. ${ }^{35}$

\section{EXPERIMENTAL RESULTS}

\subsection{Antidepressant agents}

Solutions of CIT, VEN and CBZ were prepared at different concentrations and measured both by deep-UV and visible light (Fig. 5). Deep-UV spectrum of CIT presents two peaks: (1) $1236 \mathrm{~cm}^{-1}$ and (2) $2232 \mathrm{~cm}^{-1}$, assigned to the aromatic C-F stretching vibrations and the triple-bonded C-N stretching mode, respectively. ${ }^{42}$ The waterrelated mode appears at about $1620 \mathrm{~cm}^{-1}$. The visible spectrum presents a huge fluorescence background, which prevents any reliable peak identification. The estimated LOD for the deep-UV Raman is $5 \mathrm{mg} / \mathrm{l}(12 \mu \mathrm{M})$. VEN deep-UV spectra have a slightly smaller signal to noise ratio than the CIT ones. A Raman mode is clearly identified at $1280 \mathrm{~cm}^{-1}$ and assigned to a $\mathrm{O}-\mathrm{CH}_{3}$ stretching mode. ${ }^{43}$ The visible range Raman spectrum presents a weaker fluorescence background than in case of CIT, however any clear peak identification is still perturbed by the overlap of the signals. The estimated LOD, according to a concentration series, is $6 \mathrm{mg} / \mathrm{l}(20 \mu \mathrm{M})$.

CBZ specrum is more complex, as several vibrational modes are detected. Main modes are assigned as follows: (1) $(\mathrm{C}-\mathrm{H})$ in plane bending mode at $1118 \mathrm{~cm}^{-1},(2)(\mathrm{C}-\mathrm{H})$ bending mode at $1160 \mathrm{~cm}^{-1}$, (3) (C-C) stretching modes at $1219 \mathrm{~cm}^{-1}$, (4) (C-H) bending and (C-C) stretching modes $1491 \mathrm{~cm}^{-1}$, (5) (C-C) stretching mode 1566 $\mathrm{cm}^{-1}$ and (6) (C-C) double bond stretching mode $1622 \mathrm{~cm}^{-1} \cdot{ }^{44,45}$ The background intensity of the deep-UV spectra decrease more evidently relative to the other two pharmaceuticals, as the fluorescence observed in visible Raman is weaker. The estimated LOD of CBZ is $12.5 \mathrm{mg} / \mathrm{l}(50 \mu \mathrm{M})$.

As expected, all measured compounds present no fluorescence-related background in deep-UV Raman. ${ }^{18,22}$ However, another factor, the analyte concentration, affects the background intensity, as shown prominently in the CBZ case. The dependence between the band intensity and concentration is not strictly linear for CIT and VEN, and is almost constant for CBZ (Fig. 6). At the same time, a strong attenuation of the O-H water modes at $3440 \mathrm{~cm}^{-1}$ is observed (Figs. S2, S3 and S4, Supplemental Material). Addressing such effects to self-absorption, we apply eq.(8) to estimate the absorption length $l$. UV absorption at the laser wavelength and at $\lambda=257 \mathrm{~nm}$ (O-H water mode wavelength) was measured (Fig. S5, Supplemental Material) for the following solutions: CIT (100 mg/l), VEN (50 mg/l), CBZ (33 mg/l). The estimated $l(\mathrm{~mm})$ values and all the coefficients are listed in Table S1 (Supplemental Material). The strongest inhibition of the water mode is observed in CBZ, where the highest absorption is also measured. The estimated $l=9.8 \mathrm{~mm}$ is as long as the cuvette $(10 \mathrm{~mm})$. This physically means that the beam is extensively absorbed over the entire illuminated volume $\mathrm{V}^{41}$ explaining the observed non-linearity that originates from absorption (Fig. 6). CIT presents the lowest absorptivity, the highest $k_{i}$ ratio and $l=6.7 \mathrm{~mm}$.

\subsection{Nitrate and nitrite}

Compared to visible Raman spectra, the deep-UV Raman of a $100 \mathrm{mg} / 1 \mathrm{NaNO}_{3}$ solution (Fig. 7 top), exhibits a clearly visible $\mathrm{NO}_{3}^{-}$peak at $1050 \mathrm{~cm}^{-1}$, which corresponds to the $\nu$ symmetric stretching mode. The related LOD, as demonstrated in our previous study, ${ }^{31}$ is approximately $2 \mathrm{mg} / \mathrm{l}$ and it is achievable by simply increasing the integration time from 20 to 60 seconds. Notably, a broader Raman mode at around $1330 \mathrm{~cm}^{-1}$ is assigned to a $\mathrm{NO}_{2}^{-}$vibration. ${ }^{30,31}$ As discussed in our previous work, for $\lambda_{e x c}=236.5 \mathrm{~nm}$ a quantum yield $\phi=0.05$ was estimated, hence a contribution of nitrite within the LOD is not surprising. The visible spectrum has lower signal to noise ratio. Above the fluorescence background only water-related bands at $1620 \mathrm{~cm}^{-1}$ and about 2100 $\mathrm{cm}^{-1}$ are detectable, with only a minor, barely visible nitrate band at $1050 \mathrm{~cm}^{-1}$. Nitrate was clearly detectable also in the samples taken from an aquatic ecosystem (Fig.7 bottom), including a $\mathrm{NO}_{2}^{-}$peak indicative of $\mathrm{NO}_{3}^{-}$ photolysis. Using the intensity of the $\nu$ nitrate peak in the lab-made solution as a reference, an estimated 35 $\mathrm{mg} / \mathrm{l} \mathrm{NO}_{3}^{-}$is present in the river. These results agree with the Ion Chromatography (IC) analysis adopted as a validation method. The latter estimated a $25 \pm 0.04 \mathrm{mg} / \mathrm{l} \mathrm{NO}-$ concentration, with no presence of nitrite (at a concentration higher than $0.1 \mathrm{mg} / \mathrm{l}$ ). The fluorescence background of visible Raman is especially strong in the river water spectrum, more than a factor of ten compared to the pure nitrate solution. We ascribe it 

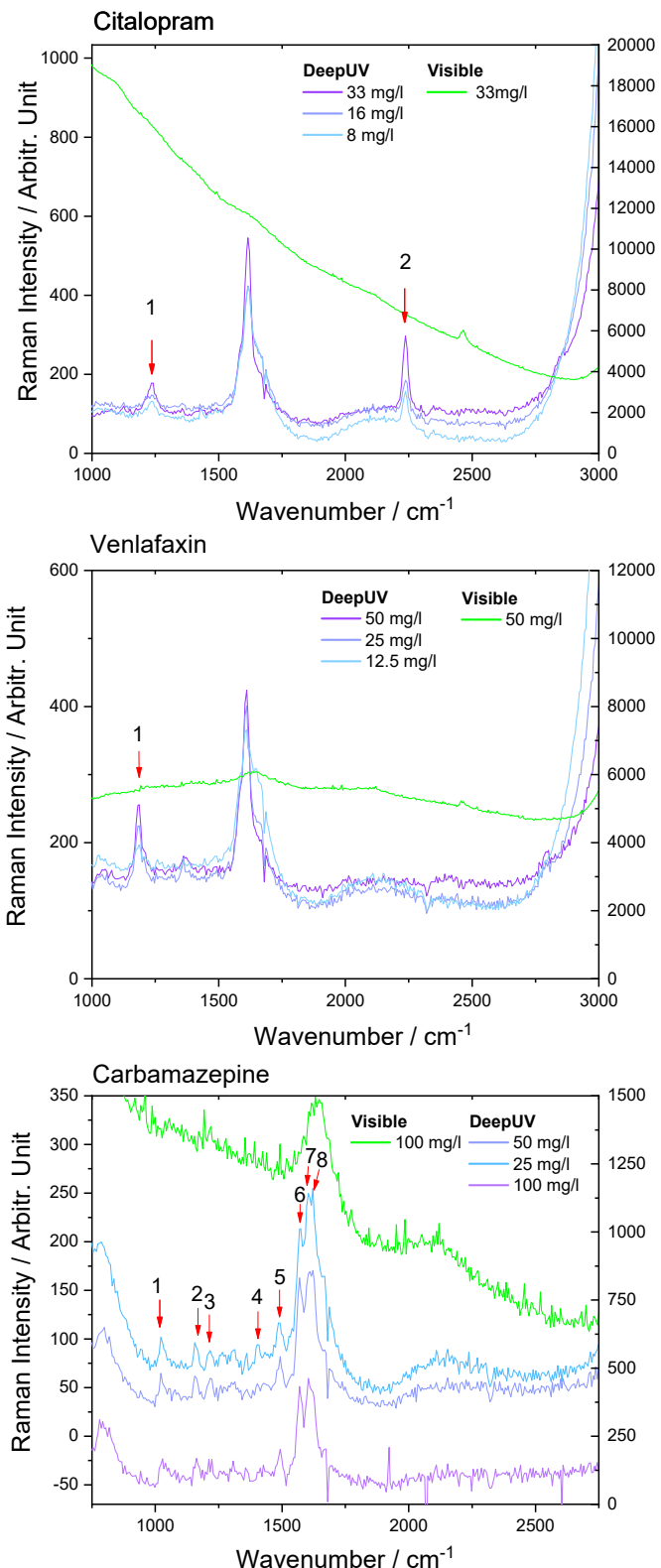

Figure 5. Deep-UV and visible Raman spectra of CIT, VEN, CBZ at different concentrations. Main Raman modes are marked and assigned in the text. The left and right axes refer to the deep-UV and visible Raman measurements, respectively. The constant background is arbitrary and here used to increase readability of the spectra.

to the presence of dissolved organic matter. ${ }^{46}$ Therefore, the visible Raman, unlike its deep-UV alternative, is unsuitable for the detection of environmentally-relevant nitrate concentrations, especially in the samples taken directly from aquatic ecosystems. It can be noted, that the $\mathrm{O}-\mathrm{H}$ water peak is slightly suppressed compared to the Raman spectra of the pharmaceuticals. By applying the same procedure as outlined above, the absorption length for the river sample (Table S1, Supplemental Material) was evaluated. Notably, the beam is absorbed within a limited length $(l=2.4 \mathrm{~mm})$, hence the intensity of the nitrate mode has to scale linearly with the concentration, as predicted in the section 3.2. UV Absorption spectra are plot in Fig. S6 (Supplemental Material). The relatively high molar absorptivity $\alpha$ is assigned to the presence of organic matter. 


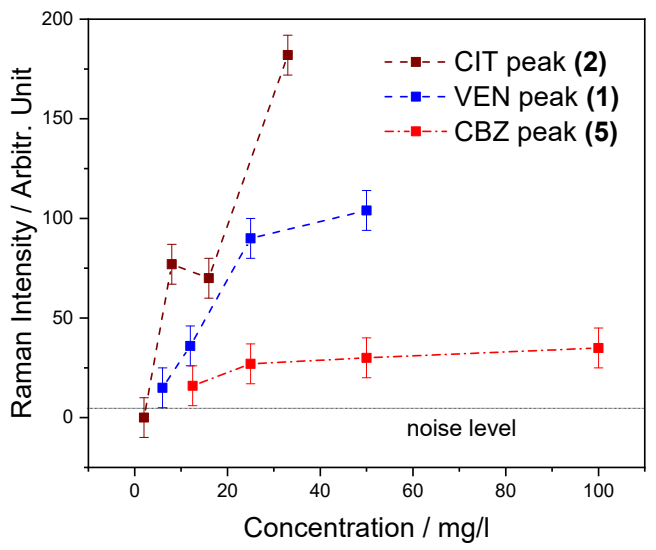

Figure 6. Intensity of CIT(2)@ $2232 \mathrm{~cm}^{-1}, \operatorname{VEN}(1) @ 1280 \mathrm{~cm}^{-1}$ and CBZ(5)@1566 $\mathrm{cm}^{-1}$ Raman modes as a function of the concentration. The noise level is marked, (approximately 5 counts arbitrary unit).
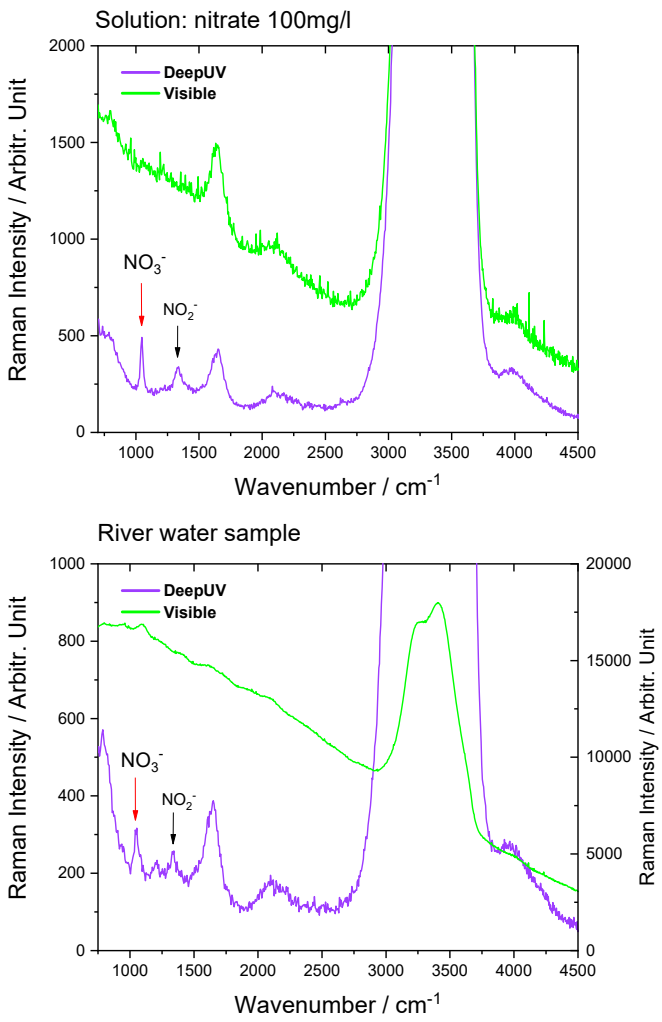

Figure 7. Deep-UV and visible Raman measurements of: (top) $100 \mathrm{mg} / \mathrm{l} \mathrm{NaNO}_{3}$ solution and (bottom) of the river water sample. The main features are marked by a red and black arrows for $\mathrm{NO}_{3}^{-}$and $\mathrm{NO}_{2}^{-}$, respectively.

\subsection{Benzotriazole}

Owing to its low sensitivity, visible Raman is not commonly used for detecting benzotriazole in solution at low concentrations $(\mathrm{mg} / \mathrm{l})$. On the other hand, the use of deep-UV light is hugely hindered by the ultraviolet absorption exhibited by this compound. ${ }^{8}$ In fact, the molecule presents two main absorption peaks at 259 and 278 nm. ${ }^{47}$ Aware of such limitations, we first evaluated visible Raman LOD by performing a high concentration series (from $500 \mathrm{mg} / \mathrm{l}$ to $10 \mathrm{~g} / \mathrm{l}$ (Fig. S7, Supplemental Material). Despite the presence of fluorescence background between 500 and $2500 \mathrm{~cm}^{-1}$, main benzene ring modes are detectable at 783, 1010 and $1387 \mathrm{~cm}^{-1}$. As expected, the minimal concentration for reliably identifying those vibrational modes is high, approximately $1 \mathrm{~g} / \mathrm{l}$. The 

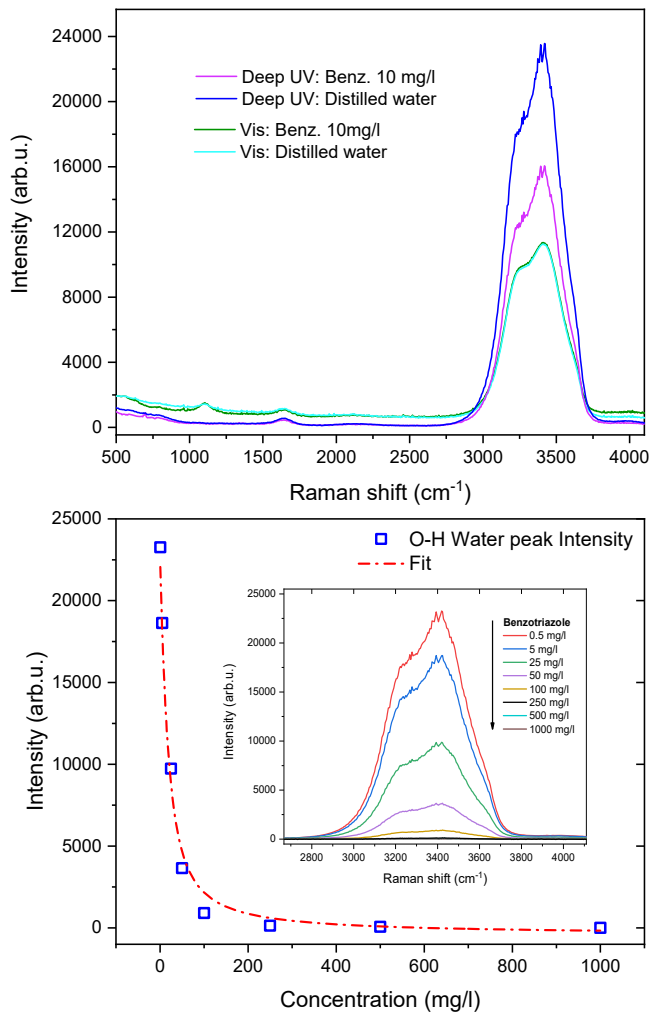

Figure 8. (Top) Deep UV and visible measurement of deionized water and $10 \mathrm{mg} / \mathrm{l}$ benzotriazole concentration solution. (Bottom) Water Raman mode intensity (O-H stretching water mode at $3420 \mathrm{~cm}^{-1}$ ) plotted with respect to benzotriazole concentration and fitted through eq.(9). Inset: corresponding spectra (0.5 mg/l, $5 \mathrm{mg} / \mathrm{l}, 25 \mathrm{mg} / \mathrm{l}, 50 \mathrm{mg} / 1,100 \mathrm{mg} / \mathrm{l}, 250$ $\mathrm{mg} / \mathrm{l}, 500 \mathrm{mg} / \mathrm{l}, 1000 \mathrm{mg} / \mathrm{l}$ concentration).

deep-UV Raman concentration series were performed on a different range, from 0.5 to $1000 \mathrm{mg} / \mathrm{l}$. As before, no fluorescence overlap was present, however, unexpectedly, no benzotriazole related Raman modes were identified at any concentrations (see Fig. S8 Supplemental Material). Furthermore, above $1 \mathrm{~g} / \mathrm{l}$ all Raman modes disappear and almost no signal is acquired. This effect of attenuation is most evident on the main water O-H modes at $3420 \mathrm{~cm}^{-1}$ (Fig. 8 top), with a strong attenuation in the deep-UV range and virtually none in the visible. This absorption effect is quantitatively and exponentially correlated to the analyte concentration (Fig. 8 bottom), with an appreciable drop above approximately $0.5 \mathrm{mg} / \mathrm{l}$ concentrations. Fitting the experimental data to the self absorption model discussed above yields the following function, which is based on eq.(8):

$$
I(x)=A\left(\frac{1-e^{-C x}}{C x}\right)+B
$$

where the constant $A=I_{0}, x$ is the independent variable, $\mathrm{C}$ and $\mathrm{B}$ are free fit parameters. The intensity of the water peak is well fitted by the model $\left(R^{2}=0.99\right.$ ), (Fig. 8 bottom), providing a value of $C_{f i t}=0.088$. This term represents the main calibration parameter for estimating the analyte concentration, while B accounts for intensity of the signal background. We notice that few data points (from 50 to $250 \mathrm{mg} / \mathrm{l}$ ) lie below the fitting curve and this can be ascribed to a variation of the laser intensity during the measurement process. The penetration depth, calculated for a $50 \mathrm{mg} / \mathrm{l}$ solution, is equal to $7.8 \mathrm{~mm}$ (Table S1, Supplemental Material), indicating an extensive beam absorption.

\section{FUTURE PERSPECTIVES}

In this paper, various potent pollutant have been successfully measured, although the achieved LOD is much above their average concentration in urban waste water (tens of $\mu \mathrm{g} / \mathrm{l}$ ). Briefly, we summarize in this sections the 
main outcomes and our strategies to improve current limitations.

\subsection{Photolysis}

Nitrate was successfully detected in pure water $(2 \mathrm{mg} / \mathrm{l})$ and in aquatic ecosystem, and the LOD may be improved as discussed above. In particular the deep-UV Raman analysis of nitrate in aquatic ecosystems is promising, as dissolved organic matter often generates an overlapping fluorescence background, which hinders the visible or near-UV Raman analysis. Nevertheless, the photolysis of nitrate ions upon UV light illumination affects the reliability of the analysis. The photolysis effect is diminished by the reduction of the exposure time of the sample to UV light, e.g. by means of a circulating liquid cell that allows for a "constant flow" mode measurement.

\subsection{Absorbance}

Benzotriazole vibrational modes were not detected using deep-UV light at any concentration between $0.5 \mathrm{mg} / \mathrm{l}$ to $1 \mathrm{~g} / \mathrm{l}$, due to the self-absorption phenomenon. This effect highlights the constraint for a potential use of deep-UV Raman spectroscopy as an environmental analysis method. If a strong UV absorber is present in the specimen, the light may be completely attenuated by the UV absorbing compounds. However, using the Raman intensity of water as internal standard it is possible to estimate the concentration of the UV-absorbers in the solution even in the absence of Raman signal from the analyte. Pure water can be used for reference. This can be achieved in one set-up, e.g by using a liquid cell connected to both the solution and the pure water. The measured Raman intensities of detected pollutants can then be corrected using the model introduced in the theory section. The intensity change on the water signal is a qualitative indicator of the overall water purity comparable to already established UV-absorption sensors with the advantage of providing fundamental chemical information not accessible otherwise. In particular for automatic sensing applications, this quality factor is of high relevance. This is most easily be explained using an example. The Raman setup will have difficulties in detecting nitrate, if the solution also contains benzotriazol. As benzotriazol (at these concentrations) does not show Raman lines, an automatic system will report 'clean water'. The quality factor using the water Raman signal does not provide a chemical analysis, but ensures that the Raman signal is reliable in case of no changes. Small changes can be used to correct the Raman analysis, while strong changes indicate that the Raman analysis is not reliable anymore.

\subsection{Estimation of minimal limit of detection}

The above given concrete examples may generalized to estimate the theoretically minimal limit of detection. We have to consider:

- wavelength selection: the water window ends around $200 \mathrm{~nm}$, the chosen wavelength of $236 \mathrm{~nm}$ is nearly ideal. ${ }^{17}$ At this wavelength, the absolute Raman crosssections for water and archetypal organic substances such as ethanol are similar. ${ }^{48}$ Although resonance enhancement may be possible, the wavelength dependence is negligible in the targeted range (deepUV). ${ }^{49}$

- signal-to-noise ratio: peaks outside the water Raman transitions are detectable, if their signal is above the noise of background signal from water, which is estimated to be 100 to 1000 times lower than the Raman transitions (see, e.g., Figs. 8, 5), i.e., the signal intensity $I_{s}$ must be greater than

$$
I_{s}>S N R \frac{I_{\text {water }}}{1000}
$$

The ratio $I_{s} / I_{\text {water }}$ is given the Raman crosssections times concentration, which are assumed to be equal (see above):

$$
\frac{I_{s}}{I_{\text {water }}}=c
$$

The minimal detectable concentration is thus simply

$$
c_{m}=\frac{S N R}{1000}
$$

For environmental water analysis, a limit of detection in the order of $\mu \mathrm{g} / \mathrm{l} \simeq c_{m}=10^{-12}$ is envisioned. To reach this value, an SNR of $90 \mathrm{~dB}$ must be achieved. 
- Increasing SNR: various advances are required to achieve an SNR of $90 \mathrm{~dB}$. This includes adopting collection optics of shorter focal length and higher numerical aperture (N.A.). ${ }^{50}$ Actually, in our setup the N.A. is relatively small $(\approx 0.3)$ with respect to an average commercial Raman spectrometer. UV compatible lenses with high N.A. are commercially available. Taking as example (Edmund Optics) a fused-silica aspherical lens with N.A. $=0.50$ a significant increase in the intensity is expected. By assuming that the radiant flux collected by the optical component is $\phi \propto(F / \#)^{2}$ and being the N.A. $=\frac{1}{(2 N . A .)}$, the collected light can be increased by at least 4 times. By reducing the focal length the beam waist decreases accordingly, minimizing l. Further signal to noise improvements are also achievable by using a highly sensitive spectrometer, such as an intensified CCD sensor (ICCD-based detector), increasing intensity by another factor of 10 to 1000 . However, marked increase of the laser beam intensity is not advisable because of the associated photolysis effects. Although the SNR is only proportional to the square root of intensity increase, the envisioned limit of detection is achievable.Furthermore, the use of a reference cell would allow easy subtraction of the reference spectrum from the investigated one by alternating measurement of the two samples, thus further increasing the sensitivity of the analysis. Time-resolved approaches, e.g. time-gated Raman (TG Raman), would be a valid technique to be considered to reduce the fluorescence while increasing the SNR ration. During last years the diffusion of this technique has been boosted through different application fields due to remarkable improvements in the electronics, laser sources and sensing. ${ }^{51}$

\section{CONCLUSIONS}

Opportunities and drawbacks of deep-UV Raman spectroscopy for monitoring water quality have been outlined by benchmarking a cost-effective and portable deep-UV Raman spectrometer. The outlined system is based on a commercial deep-UV laser source and inexpensive optical components. Potentially harmful compounds were measured at environmentally relevant concentrations in pure water and in aquatic environment, providing a reliable LOD for the technique. In particular, the impact of fluorescence, photolysis and self-absorption during the analysis process is thoroughly discussed. Such effects, can be considered as collateral since they invalidate the measurement by affecting the Raman spectra. Adopting a simple mathematical model, the self-absorption was described and the beam absorption depth quantified. In principle, by implementing the various measures including improvement of hardware as well as evaluation procedures, it is foreseeable that an LOD in the $\mu \mathrm{g}$ range is reachable, being an important benchmark in environmental water analysis. The perspective to carry out Raman spectrometry using narrow line X-ray pulses (see also Sects. 1-4 [52]) on electronic transition is attractive.

\section{ACKNOWLEDGMENTS}

The project was partially funded by BAFU (Bundesamt für Umwelt). We thank Renato Figi and Jasmin Hubeli for IC measurements performed at Empa.

\section{REFERENCES}

[1] "Clean water and sanitation." https://www.un.org/sustainabledevelopment/water-and-sanitation/.

[2] Singh, B. and Sekhon, G., "Nitrate pollution of groundwater from farm use of nitrogen fertilizers - a review," Agriculture and Environment 4, 207-225 (Mar. 1979).

[3] Doane, T. A. and Horwáth, W. R., "Spectrophotometric determination of nitrate with a single reagent," Analytical Letters 36, 2713-2722 (Jan. 2003).

[4] Trček, B., Žigon, D., Zidar, V. K., and Auersperger, P., "The fate of benzotriazole pollutants in an urban oxic intergranular aquifer," Water Research 131, 264-273 (Mar. 2018).

[5] Lajeunesse, A., Smyth, S., Barclay, K., Sauvé, S., and Gagnon, C., "Distribution of antidepressant residues in wastewater and biosolids following different treatment processes by municipal wastewater treatment plants in canada," Water Research 46, 5600-5612 (Nov. 2012).

[6] Giebultowicz, J. and Nalecz-Jawecki, G., "Occurrence of antidepressant residues in the sewage-impacted vistula and utrata rivers and in tap water in warsaw (poland)," Ecotoxicology and Environmental Safety 104, 103-109 (June 2014). 
[7] Schultz, M. M., Furlong, E. T., Kolpin, D. W., Werner, S. L., Schoenfuss, H. L., Barber, L. B., Blazer, V. S., Norris, D. O., and Vajda, A. M., "Antidepressant pharmaceuticals in two u.s. effluent-impacted streams: Occurrence and fate in water and sediment, and selective uptake in fish neural tissue," Environmental Science 83 Technology 44, 1918-1925 (Mar. 2010).

[8] Molins-Delgado, D., Távora, J., Díaz-Cruz, M. S., and Barceló, D., "UV filters and benzotriazoles in urban aquatic ecosystems: The footprint of daily use products," Science of The Total Environment 601-602, 975-986 (Dec. 2017).

[9] Ward, M., Jones, R., Brender, J., de Kok, T., Weyer, P., Nolan, B., Villanueva, C., and van Breda, S., "Drinking water nitrate and human health: An updated review," International Journal of Environmental Research and Public Health 15, 1557 (July 2018).

[10] Wick, K., Heumesser, C., and Schmid, E., "Groundwater nitrate contamination: Factors and indicators," Journal of Environmental Management 111, 178-186 (Nov. 2012).

[11] Assubaie, F. N., "Comparative study of methods used for the determination of nitrate and nitrite pollutants in well, ground water and soil in al-hassa area, saudi arabia," Pakistan Journal of Biological Sciences 7, 508-513 (Apr. 2004).

[12] Goldstein, S. and Rabani, J., "Mechanism of nitrite formation by nitrate photolysis in aqueous solutions: the role of peroxynitrite, nitrogen dioxide, and hydroxyl radical," Journal of the American Chemical Society 129, 10597-10601 (June 2007).

[13] Meusinger, C., Berhanu, T. A., Erbland, J., Savarino, J., and Johnson, M. S., "Laboratory study of nitrate photolysis in antarctic snow. i. observed quantum yield, domain of photolysis, and secondary chemistry," The Journal of Chemical Physics 140, 244305 (June 2014).

[14] Sharpless, C. M. and Linden, K. G., "UV photolysis of nitrate: effects of natural organic matter and dissolved inorganic carbon and implications for UV water disinfection," Environmental Science $\mathcal{E}$ Technology 35, 2949-2955 (July 2001).

[15] Ludovici, M., Ialongo, C., and Camera, E., "Principles, current applications, and future perspectives of liquid chromatography-mass spectrometry in clinical chemistry," in [Liquid Chromatography], 727-751, Elsevier (2017).

[16] Hundt, P. M., Tuzson, B., Aseev, O., Liu, C., Scheidegger, P., Looser, H., Kapsalidis, F., Shahmohammadi, M., Faist, J., and Emmenegger, L., "Multi-species trace gas sensing with dual-wavelength qcls," Applied Physics B: Lasers and Optics 124(6), 108 (2018).

[17] Hale, G. M. and Querry, M. R., "Optical constants of water in the 200-nm to 200- $\mu$ m wavelength region," Appl. Opt. 12, 555-563 (Mar 1973).

[18] Kostamovaara, J., Tenhunen, J., Kögler, M., Nissinen, I., Nissinen, J., and Keränen, P., "Fluorescence suppression in raman spectroscopy using a time-gated CMOS SPAD," Optics Express 21, 31632 (Dec. 2013).

[19] Bruzas, I., Lum, W., Gorunmez, Z., and Sagle, L., "Advances in surface-enhanced raman spectroscopy (SERS) substrates for lipid and protein characterization: sensing and beyond," The Analyst 143(17), 39904008 (2018).

[20] Demirel, G., Usta, H., Yilmaz, M., Celik, M., Alidagi, H. A., and Buyukserin, F., "Surface-enhanced raman spectroscopy (SERS): an adventure from plasmonic metals to organic semiconductors as SERS platforms," Journal of Materials Chemistry C 6(20), 5314-5335 (2018).

[21] Tolles, W. M., Nibler, J. W., McDonald, J. R., and Harvey, A. B., "A review of the theory and application of coherent anti-stokes raman spectroscopy (CARS)," Applied Spectroscopy 31, 253-271 (July 1977).

[22] Asher, S. and Johnson, C., "Raman spectroscopy of a coal liquid shows that fluorescence interference is minimized with ultraviolet excitation," Science 225, 311-313 (July 1984).

[23] Troyanova-Wood, M. A., Petrov, G. I., and Yakovlev, V. V., "Simple and inexpensive instrument for deepUV raman spectroscopy," Journal of Raman Spectroscopy 44, 1789-1791 (Oct. 2013).

[24] Asher, S. A., "UV resonance raman spectroscopy for analytical, physical, and biophysical chemistry. part 1," Analytical Chemistry 65, 59A-66A (Jan. 1993).

[25] Efremov, E. V., Ariese, F., and Gooijer, C., "Achievements in resonance raman spectroscopy," Analytica Chimica Acta 606, 119-134 (Jan. 2008). 
[26] Domes, C., Domes, R., Popp, J., Pletz, M. W., and Frosch, T., "Ultrasensitive detection of antiseptic antibiotics in aqueous media and human urine using deep UV resonance raman spectroscopy," Analytical Chemistry 89, 9997-10003 (Aug. 2017).

[27] Ianoul, A., Coleman, T., and Asher, S. A., "Uv resonance raman spectroscopic detection of nitrate and nitrite in wastewater treatment processes," Analytical Chemistry 74, 1458-1461 (Mar. 2002).

[28] Furuya, N., Matsuyuki, A., Higuchi, S., and Tanaka, S., "Determination of nitrate ion in waste and treated waters by laser raman spectrometry," Water Research 13(4), 371-374 (1979).

[29] Gajaraj, S., Fan, C., Lin, M., and Hu, Z., "Quantitative detection of nitrate in water and wastewater by surface-enhanced raman spectroscopy," Environmental Monitoring and Assessment 185, 5673-5681 (Oct. 2012).

[30] Asher, S. A., Tuschel, D. D., Vargson, T. A., Wang, L., and Geib, S. J., "Solid state and solution nitrate photochemistry: Photochemical evolution of the solid state lattice," The Journal of Physical Chemistry A 115, 4279-4287 (May 2011).

[31] Sterzi, A., Schneider, U., Sambalova, O., Bleiner, D., and Borgschulte, A., "Tunable deep-UV raman spectroscopy reveals nitrate photolysis," in [UV and Higher Energy Photonics: From Materials to Applications 2019], Lérondel, G., Cho, Y.-H., Kawata, S., and Taguchi, A., eds., SPIE (Sept. 2019).

[32] Bykov, S. V., Mao, M., Gares, K. L., and Ashera, S. A., "Compact solid-state $213 \mathrm{~nm}$ laser enables standoff deep ultraviolet raman spectrometer: Measurements of nitrate photochemistry," Applied Spectroscopy 69, 895-901 (Aug. 2015).

[33] Lednev, I. K., Karnoup, A. S., Sparrow, M. C., and Asher, S. A., "-helix peptide folding and unfolding activation barriers: a nanosecond UV resonance raman study," Journal of the American Chemical Society 121, 8074-8086 (Sept. 1999).

[34] Gares, K. L., Hufziger, K. T., Bykov, S. V., and Asher, S. A., "Review of explosive detection methodologies and the emergence of standoff deep UV resonance raman," Journal of Raman Spectroscopy 47, 124-141 (Jan. 2016).

[35] Hollis, J. R., Rheingold, D., Bhartia, R., and Beegle, L. W., "An optical model for quantitative raman microspectroscopy," Applied Spectroscopy 74, 684-700 (Apr. 2020).

[36] D’Amico, F., Saito, M., Bencivenga, F., Marsi, M., Gessini, A., Camisasca, G., Principi, E., Cucini, R., Di Fonzo, S., Battistoni, A., Giangrisostomi, E., and Masciovecchio, C., "Uv resonant raman scattering facility at elettra," Nuclear Instruments and Methods in Physics Research Section A: Accelerators, Spectrometers, Detectors and Associated Equipment 703, 33-37 (2013).

[37] Masciovecchio, C., Bencivenga, F., and Gessin, A., "Water dynamics at the nanoscale," Condensed Matter Physics .

[38] "Quantum mechanical theory of rayleigh and raman scattering," in [The Raman Effect], 49-84, John Wiley \& Sons, Ltd.

[39] Hong, Z. and Asher, S. A., "Dependence of raman and resonance raman intensities on sample selfabsorption," Applied Spectroscopy 69, 75-83 (Jan. 2015).

[40] Shriver, D. F. and Dunn, J. B. R., "The backscattering geometry for raman spectroscopy of colored materials," Applied Spectroscopy 28, 319-323 (July 1974).

[41] Liu, C. and Berg, R. W., "Nonlinearity in intensity versus concentration dependence for the deep UV resonance raman spectra of toluene and heptane," Applied Spectroscopy Reviews 48, 425-437 (July 2013).

[42] [Structure Determination of Organic Compounds], Springer Berlin Heidelberg (2009).

[43] Bock, P., Nousiainen, P., Elder, T., Blaukopf, M., Amer, H., Zirbs, R., Potthast, A., and Gierlinger, N., "Infrared and raman spectra of lignin substructures: Dibenzodioxocin," Journal of Raman Spectroscopy (Jan. 2020).

[44] Czernicki, W. and Baranska, M., "Carbamazepine polymorphs: Theoretical and experimental vibrational spectroscopy studies," Vibrational Spectroscopy 65, 12-23 (Mar. 2013).

[45] Zanchi, C., Lucotti, A., Tommasini, M., Trusso, S., de Grazia, U., Ciusani, E., and Ossi, P. M., "Laser tailored nanoparticle arrays to detect molecules at dilute concentration," Applied Surface Science 396, 1866-1874 (Feb. 2017). 
[46] Aziz, F., Qazzani, N., Mandi, L, Assaad, A., Pontvianne, S., Poirot, H and Pons, M.-N., "Characteriza-tion by fluorescence of dissolved organic ratter in rural drinking water s torage tanks in 1⿴rocco," Journal of Witer and Health 16, 321-328 (Feb. 2018).

[47] Li, Y, awg, J., Guo, .MGao, C., and Cheng, Z., "Determination of benzotriazole in industrial cooling water by fluorescence," Instrumentation Science ESTechnology 45, 290-300 (Aug. 2016).

[48] Abe, N. and I to, M., "Effects of hydrogen bonding on the raman intensities of rethanol, ethanol and water," Journal of Raman Spectroscopy 7(3), 161-167 (1978).

[49] Faris, G. W. and Copeland, R. A., "Wavelength dependence of the raman cross section for liquid water," Appl. Opt. 36, 2686-2688 (Apr 1997).

[50] Kelly, J. F., Blake, TA., Bernacki, B. E., and Johnson, TJ., "Design considerations for a portable raman probe spectrometer for field forensics," International Journal of Spectroscopy 2012, 1-15 (Sept. 2012).

[51] Kögler, M. and Heilala, B., "Time-gated raman spectroscopy - a review," Measurement Science and Technology 32, 012002

[52] D. Bleiner, The Science and Technology of X-ray Lasers: A 2020 Update Proc. SPIE 11886, 1188602 (2021) 